\title{
RECHERCHES RÉCENTES SUR QUELQUES CONDITIONS DE VIE ET DE CROISSANCE DE LA TRUITE COMMUNE
}

\author{
(Salmo fario L.) \\ Par M. P. VIVIER, \\ Inspecteur des Forèls, \\ Directeur du Laboratoire central d'Hydrobiologie appliquée.
}

On manque encore, par trop, d'observations scientifiques sur les condilions de vie et de croissance des Poissons d'élevage; de telles observations peuvent ètre ccpendant d'une importance capitale pour la conduite d'un élevage rationnel ou pour la réussite du repeuplement.

l.es facteurs - phỵsiques (tompérature, oxygène dissous, lumière), mécaniques (dimensions des bassins), - biologiques (àge des reproductcurs - ont été depuis quelques années l'objet d'études assez nombreuses en Europe centrale; il nous a paru intéressant danalyser ici même quelques-uns de ces travaux, dans l'espoir (que les résultats obtenus ne seront pas inuliles aux Salmoniculteurs ou aux Officiers des Forèts chargés d'un service de pisciculture ou de pèche.

\section{1. - Température des cours d'eau à Truites.}

Le $D^{r}$ Vaclay Drk (1) a relevé dans le massif montagneux du Tatra (Bassin de l'Elbe et du Danube) plus d'une centaine de lempératures de rours d'cau à Truites duraut la saison d'élé (rg34-1938); ces températures sont des plus variables et dépendent évidemment des conditions atmosphérirues, de l'altitude, du climat, de la vitesse du courant et de l'ombrage des rives. L'amplitude moyenne des variations annuelles est considérable : - $10^{\circ}$ d'après Thunkman (g). dans le cours moyen, - I z̀ à $18^{\circ}$ dans le cours inférieur, là vì commence la zone à Ombre. Examinant les températures maxima ef les oscillations journalières d'été, le $\mathrm{D}^{\mathrm{r}}$ Vaclav Dik a réparti les cours d'eau à Truites en 7 catégories :

$1^{\circ}$ Cours d'eull de très hante altitude. - Ils sont situés à environ 1 .joo mètres et souvent alimentés par la fonte des neiges. La température maxima reste basse $\left(6\right.$ à $\left.10^{\circ}\right)$ et les oscillations journalières sont faibles (a à $\left.3^{\circ}\right)$.

(1) Die Sommertemperaturen der Forellenwâsser, - Arch. für Hydrobiologie Vol. XXXVII, 2, 1940, pp. 273-277.

(2) Die Binnengewásser Mitteleuropas, - Vol. I, Stuttgart, 1925, p. 59. 
?o Coms d'en de haute montagne (allitude moyenne 1.000 mètres). Les eaux en provenance de failles naissent souvent à une température rela. tivement élerée et voisine de leur température maxima $\left(\mathrm{I}^{\circ}\right)$. Lamplitude journalière reste faible $\left(3\right.$ à $\left.4^{\circ}\right)$.

$3^{\circ}$ Cours d'eau de montagne. - Ces cours d eau aux flols rapides sont souvent peu réchauffés par le soleil, en raison de leur encaissement. La

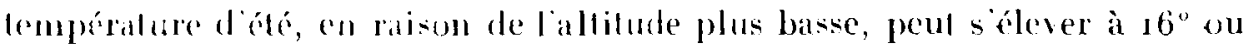
$18^{\circ}$, mais les oscillations journalières sont toujours minimes $\left(4^{\prime \prime}\right)$.

4" Cours d'eau de basses montagnes forlement ensoleillés. - La tempémalure peut s'élever, par chaudes journécs d'été, à $26^{\circ}$, mais les nuits restent fraîches el les amplitudes joumalières sont considérables $\left(10^{\circ}\right)$. A la condition, loutefois, que la dose d'oxygène dissous soit suflisante (6) centimèlres cubes par litre pour les adultes), les Truites vivent encore dans ces eaux presque chaudes; cette condition est généralement remplie

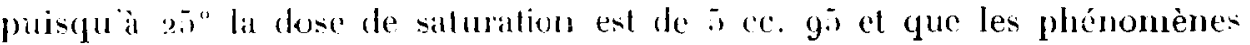
de sursaturation ne sont pas rares dans des eaux très agitées. Les Truites trousent là des conditions dexistence limite; que survienne, durant ces chaudes journées, une pollution organique, mème peu nocive par température moycnne, alors loxygène dissous tombe à des valeurs très basses it les l'oissons meurent par centaines et mème par milliers. C̈est dans ces cours deau que les industries agricoles sont le plus à craindre et c'est malheureusement là qu'elles sinstallent généralement. Il parait donc nécessaire dexiger la connaissance de la température maxima d’été du cours d'eau asant de donner une autorisation d'installation quelconque; aucune industrie agricole ne devrait ètre tolérée dans une rivière dont la tempéralure maxima dépasserail $20^{\circ}$.

5० Cours d'eau rapides el ombragés des plaleaux (altitude joo mètres). Laltilude est encore faible, mais l'ombrage apporté par l'abondante végétation des rives empèche le ruisseau de s’échaufler : la température ne dépasse guère 18 à $20^{\circ}$ à l'ombre et les oscillations journalières restent peu inlportantes $\left(4^{\circ}\right)$.

$6^{\circ}$ Cours deau pen ombragés des plateanx. - Les conditions d'altitude sont les mèmes, mais l'ombrage manque dans ces cours d'eau de pâturages, bien ensoleillés, peu profonds; les eaux s'échauffent fortement et atteignent $25^{\circ}$, les oscillations journalières devenant importantes $\left(10^{\circ}\right)$. Ce lype est très voisin du type 4.

$7^{\circ}$ Conrs d'eau froids de plaine. - L'altitude est relativement faible et le courant peu marqué ; mais les eaux sont peu ensoleillées et la température ne sélève guère plus haut que $15^{\circ}$, l Iamplitude des variations thermiques restant minime ( 3 à $4^{\circ}$ ). Les cours d'eau de ce type seraient fréquents dans le Karst.

Cette classification n'a évidemment de valeur réelle que pour la région où elle a élé stablie; néammoins elle correspond assez bien aux types de 
cours d'eau à Truites que nous avons en France : - forrents de haute altifude tels que l'Isère près de Val d’isère. - de haule montagrne comme la Dranse du Chablais, - cours d'eau de collines comme l' Iin ou de plateaux romme cem du Jura (lype 4) et des loseres (lype 5), - cours d'ean de plaine conme ceux de la Somme.

l.es précisions que l'élude apporte pourront donc itre utilement consullíes et contròlées.

\section{II. - Oxygène dissous et croissance des alevins.}

La température est, en effet. plus rapidemenl déterminable dans un cours d'eau ou un établissement de pisciculture que lo taux de l'orygène dissous : cest cependant ce facteur qui est de beaucoup le plus important, étroitement lié d’ailleurs à la température, comme chacun sait.

On admet généralement qu une cau à Truite doit conlenir de 6 à 7 renti-

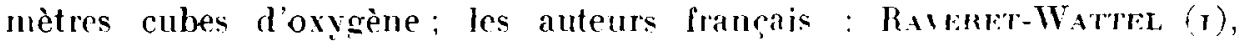
If́rar (2), Rot Le (3), ne donnent pas de valeur pour les alevins; or, ceuxci, si l'on en croit les recherches de Drwols et Wondenü̈rm (1), ont des liesoins minima en oxygène beaucoup plus faibles et que je crois intéress.nt de donner ici :

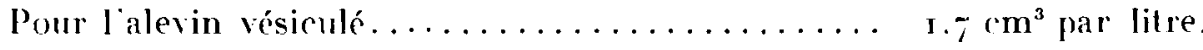

Pour l'alevin capable de s'alimenter (un peu avant

la fin de la résorbtion) $\ldots \ldots \ldots \ldots \ldots \ldots \ldots \ldots \ldots, 1,8 \mathrm{~cm}^{3}$ par litre.

Pour laler in qui salimente bicn lui-mime...... 3. $\mathrm{cm}^{3}$ par litri.

Ces doses, véritablement surprenantes, sont évidemment des doses minima, les alevins sont-ils sensibles à des teneurs plus ou moins élevées

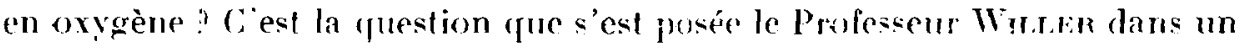
Irasail déjà ancien (i) mais qui mérite d'ìtre examiné iro :

(1) La Pisciculture, - I, Paris, p. 46.

(2) Pratique rationnelle de la petile salmoniculture fermière. - Grenoble 1936, p. 16.

(3) Manuel de Pisciculture, - Paris 1932, p. 78.

(4) Einiges über die Lebensbedingungen der Forellenbrul im Freien, -- Biolog. Zentralblatt, XLI, 1921.

J'ai arrondi les chiffre's donnés par ce's auteurs, qui comprenaient trois décimales absolument illusoires, comme je l'ai déjà montré clans : "L'oxygène dissous sous la glace ". Bulletin francais de Pisciculhure, $n^{\circ} 62$, Août 1933. - Voici d'ailleurs le texte allemand tel qu'il est rapporté par WiLLer :

"Durch die Intersuchungen von DenolL UND WomgkM T wissen wir dass der Sauerstoffbedarf der Bachforellenbrut nach dem Verlust des Dottersackes grösser wird als zuvor. - les s! arben hie'nach :

Dotterbrut bei .................

fressfaehige Brut bei. ...............

$1,692 \mathrm{~cm}^{3}$ pro 1 Liter

angefurterte Brut bei ..............

$1,803 \mathrm{~cm}^{3}$ pro 1 Liter

$3,0.19 \mathrm{~cm}^{3}$ pro 1 Liter ".

(Untersuchungen über das Wachstung bei Fischen, II, III, IV. - Weitere Untersuchungen über den Einfluss aüsserer Fahtoren auf dus Wachstum der Bach'orellenbrut. -- Zeitschrift für Fischerei, XXVI, 1928, p. 5933).

(5) Op. cit., pp. 565 à 582. 
Dans une auge 1 , paure en oxygène. il plaçait le ? Décembre rg26 des oufs fécondés le jour mìme; il en mettait une mème quantité dans une auge $B$, identique mais beaucoup plus riche. Il suivait l'évolution du peuplement dans les deux cas pendant près de cinc mois, jusqu'en Avril i $9^{2} 7$.

Les conditions étaient les suivantes :

\begin{tabular}{|c|c|c|c|}
\hline DATES & TEMPÉRATLRES & $\begin{array}{c}\text { Auge } A . \\
\text { OXYGẼE EN } \mathrm{CM}^{3} \\
\text { PAR LITRE }\end{array}$ & $\begin{array}{c}\text { Auge } B \text {. } \\
\text { OXYGìNE EN } \mathrm{CM}^{3} \\
\text { PAR IITRE }\end{array}$ \\
\hline 1er Décembre $1926 \ldots$ & $7 \circ 8$ & 2,7 & 1,4 \\
\hline 19 Janvier $1927 \ldots \ldots$ & $6^{0}$ & $3, \overline{7}$ & 6,7 \\
\hline 23 Janvier $1927 \ldots \ldots$ & 702 & 3,5 & 6,2 \\
\hline
\end{tabular}

L'embryonnement, caractérisé, comme chacun sait, par l'apparition des yeux, ne paraît pas sètre produit diff́remment dans les deux cas ; l'éclosion, par contre, commença plus tard dans l'auğe 1 et se termina plus tard comme l indique le tableau suivant :

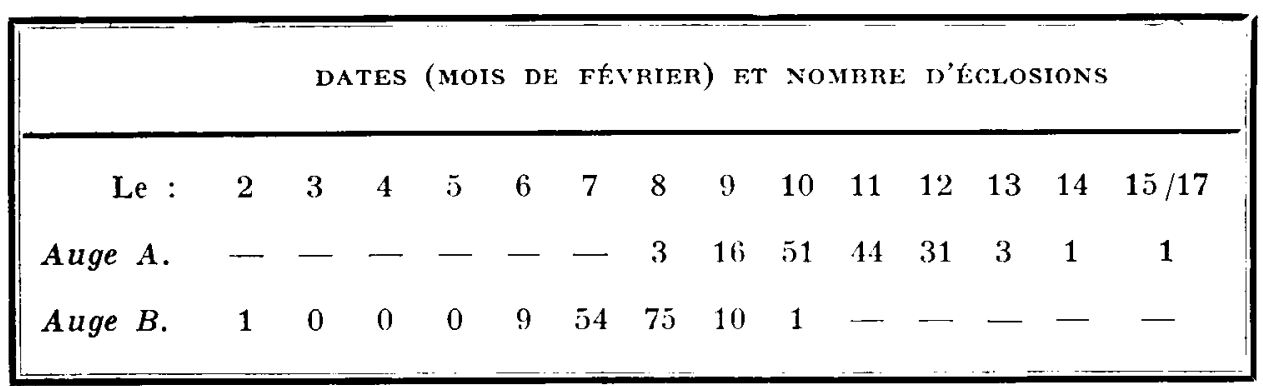

Le 12 Février 1927 , c'est-à-dire 7 ? jours après la fécondation, les alevins de l'Auge $A$ mesuraient en moxenne ri millimètres et leur poids moyen ćtait de 74,6 milligrammes; dans l'auģe B leur longueur moyenne atteignait I $_{7} \mathrm{~mm} .9^{6}$ et leur poids $80, ?$ millig̣rammes. Lc graphique ci-après (Fig. ro) met bien en évidence le rapport entre les longueurs et le nombre d'individus dans chaque auge: il fait ressortir d ume façon frappante que la forte llongueur moyenne de l'alevin de l'auge $B$ n'est pas le fait de quelques individus exceptionnellement gros, mais de l'ensemble du peuplement:

Le 22 Avril I 927 , soit 138 jours après la fécondation et 23 jours après le début de l'alimentation, la situation s'était renversée : l'ange $\mathrm{A}$, la plus pauvre en oxygène, contenait alors la plus grande quantité de gros alevins. La longueur moyenne était en effet de 25. 29 millimètres et le poids moyen de 130,97 milligrammes; dans l'auge B, la longueur moyenne ne dépassait pas 24,82 millimètres et le poids moyen $10 \$, 84$ milligrammes. Le gra- 
phique ci-dessous (Figr. I1) met parfaitement en évillence ces résultats au premier abord inattendus :

Cette modification si curicuse de croissance, en faveur d'alevins placés dans des eaux pauves en oxygène, s'explirque cependant facilement; le besoin de nourriture $y$ est moins grand que dans un milieu fortement

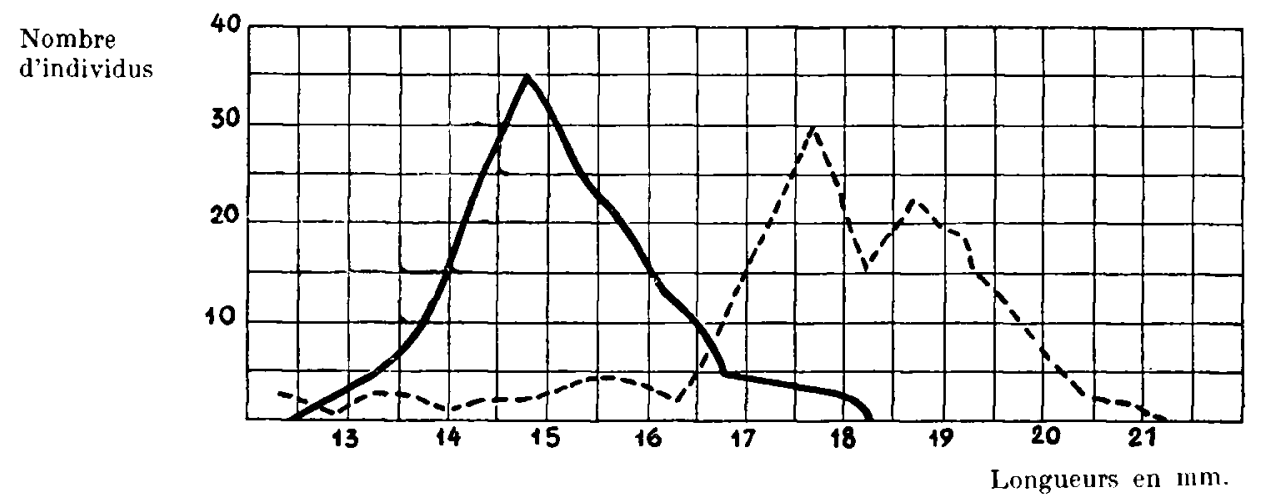

Fig. 10. - Courbe des longueurs it jours après la fécondation (d'après IVILlea).

- dans lange $A$, pauvre en oxygène.

- - - dans l'auge $b$, rijhe en oxygene.

Nombre d'individus.

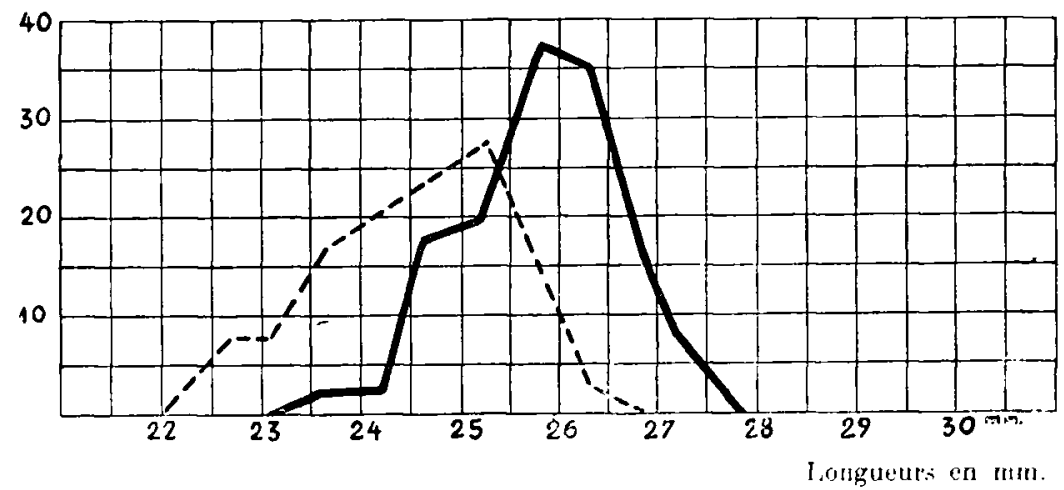

FIg. 11. - Courbe des longuenrs 138 jours après la ficondation et 23 jours apries le debut de lablimentation (d'apres Wilifa).

- _ C dans l'auge $A$, paure en oxygène.

_... dans laage $B$, riche en oxygene.

oxycrénisé; la nourriture étant restée la mime dans les deux augres, les alevins de l'auge 1 ont pu lirer un meilleur parti des aliments: mais, si I'on augmente la nourriture des alevins de lauge $B$, pour faire face aus dépenses plus vives d'énergrie, que nécessite une eau plus riche en oxygène, alors la roissance y restera de beauroup la plas forte. J'expérience tentóe l'année suivante ( 1928 ) confirma pleinement cette conclusion.

Fin résumé, en gćnćralisant les résultots précédemment indiqués et d'autres relatifs à la mortalité comparée dans les deux auges, on peut dire que l'orygène : 
I $^{\circ}$ - dimimue la montaliti (S.7 \% lans lauge 1 , contre 5,3 dans lauge B) ;

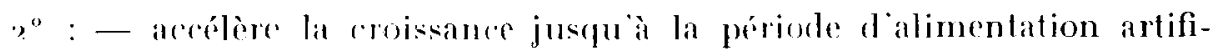
(iclle ;

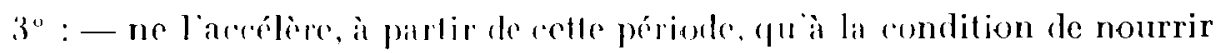
l'alevin en proportion de la dose arrerue doygène qui lui est donnée.

11 est done recommandé de donner aur ands et aur alevins une eau la plus aérée possible.

\section{III. - Lumière et croissance des alevins.}

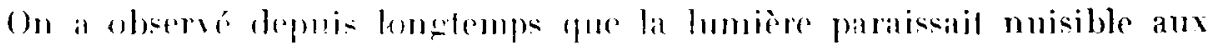
wuth et aux alevins; a cest notamment lavis de nombreux agronomes tels

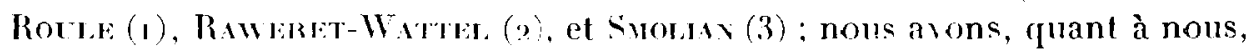
toujours exigé daus nos établisements de salmoniculture que les auges soient en partie garmies de comercles de bois. Il était rependant intéressant de connaitre lis limites de cette nocivité. ('est re qui a tenté précisément Wir.ten, en 1926 (१) :

Il plaçail, le ? Décembre 1926. des a'ufs fécondés le jour même dans deux aug̣es d'égales dimensions et alimentérs par un courant de débit normal (I.125 (entimètres cubes à la minute). L cau provenait de la mème source que dins les expériences indiquées plus haut et contenait des doses dovytrène correspondant à relles titrées dans l'auge 1 . done faibles; les conditions thermiques élaient les mêmes. L'une de ces aựes (C) élait recouverte d'un panneau de zinc bien noirci à l'asphalte; les ouufs n'étaient visites qu'à la nuit, au faible éclairage d'une petite lampe à pétrole; au contraire la deuvième augue (D) demeurait à ciel ouvert. hien exposée à la lumière. sans toutefois reccroir les rayons fmp riches en radiations ultraviolctes.

L'éclosion des alcvins se révéla plus précoce de 6 jours dans l'auge $C$, obscure : elle dura aussi plus longtemps (r) jours contre 8) :

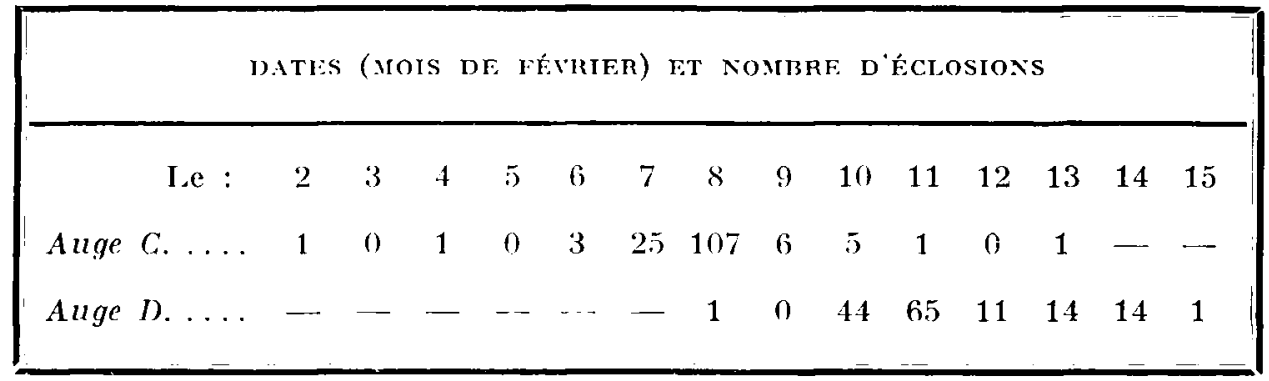

(1) Op. cit.. p. 90 .

(2) Op. cit., I. p. 105.

(3) Merkbuch der Binnenfischerei, - Berlin 1920.

(1) Op. cit. pp. is' a is ist3. 
Les alevins, à l'obscurité, apparurent de suite plus gros que les autres; lès le 19 Fónrier 19\%- 79 junts après la fécondation, ils mesuraient, dans l'auge $\mathrm{C}$, i 8,56 millimètres en moyenne et leur poids moyen était de 85,3 milligrammes; au conlräire, dans l'auge $\mathrm{D}$, la longueur ne dépassait jas 16,09 millimètres et le poids se trourait de 79,64 milligrammes; le yraphique ci-après (Fig. 12) muntre bien la différence des deux peuplements :

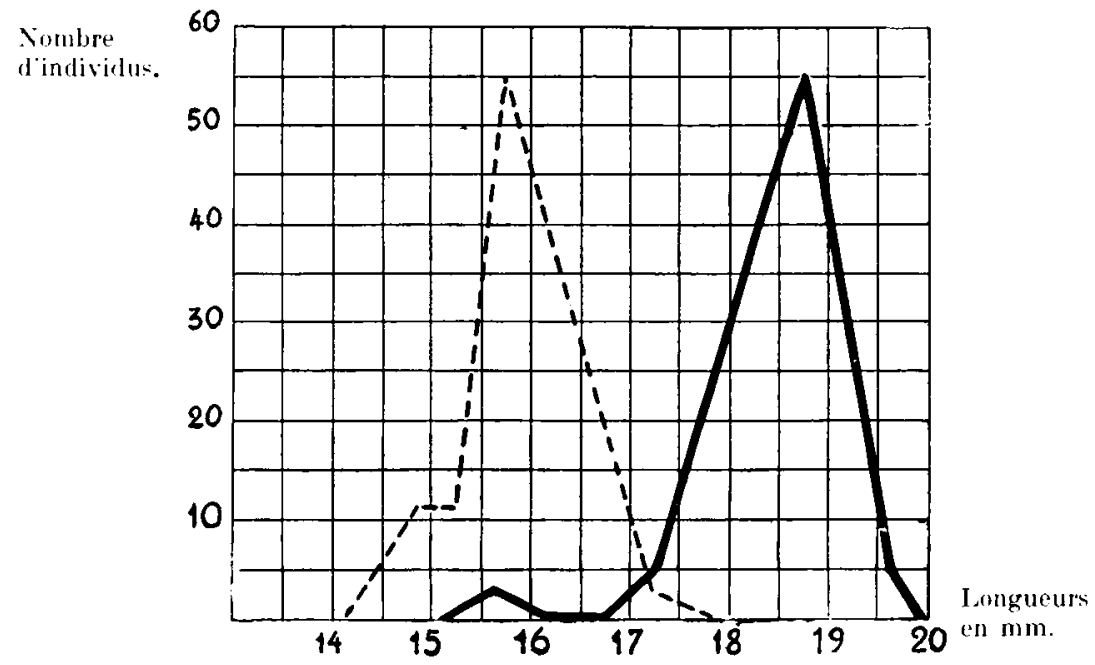

Fig. 12. - Courbe des longueurs, 79 jours apres la fécondation (d'après Willer).

- - - dans l'auge C, obscure.

- . - dans lange D, laire.

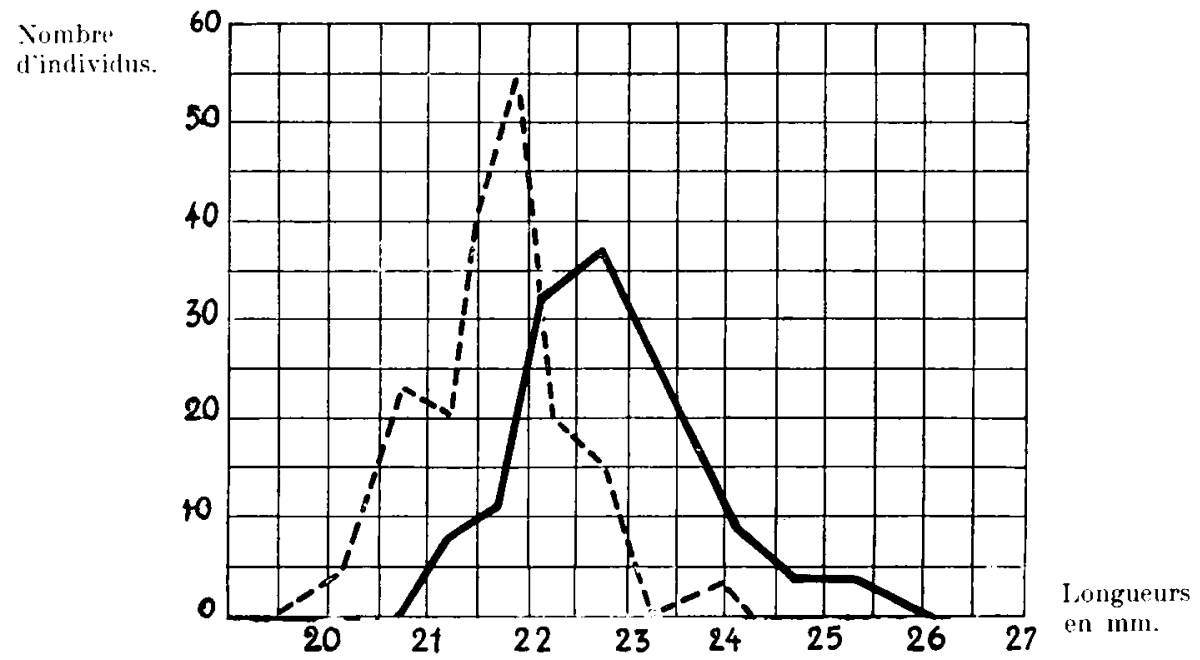

Firi. 13. - Courbe des longueurs, 105 jours apres la fécondation (d'apres Willy: ).

- dans l'auge $C$, obseure.

- -.... dans l'auge $D$, claire.

Le iz Mars I927, soit yoj jours après la fécondation, la longueur et le 
poids moyen étaient encore supérieurs dans l'auge obscure, mais les différences saltémaient; dans laume $C$, la longueur moyenne atteignait 22,9 ; millimètres ef le poids moyen roo,18 milligrammes; dans l'auge $\mathrm{D}$, la longueur ne dépassail pas 29,3 millimètres el le poids 93,68 milligrammes; le graphique di-aranl (Fiz. 13) caraclérise parfaitement cette nourclle situation :

Le 2 I Avril I9?7, soit 137 jours après la fécondation et 22 jours seulement après le commencement de l'alimentation, les résultats de l'alevinagge dans l'auge obscure et dans l'auge clitire se trouvaient tout à lait comparables; lit longueur moyenne dans l'auge (' était de $25, \overline{5} 7$ millimètres et le poids moven de r 29 , fo milligrammes; dans l'auge $D$ la longueur attei-

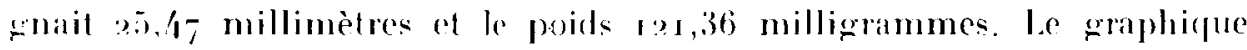
suivant (Fig. 19) montre bien la superposition des deux peuplements :

Nombre d'individus.

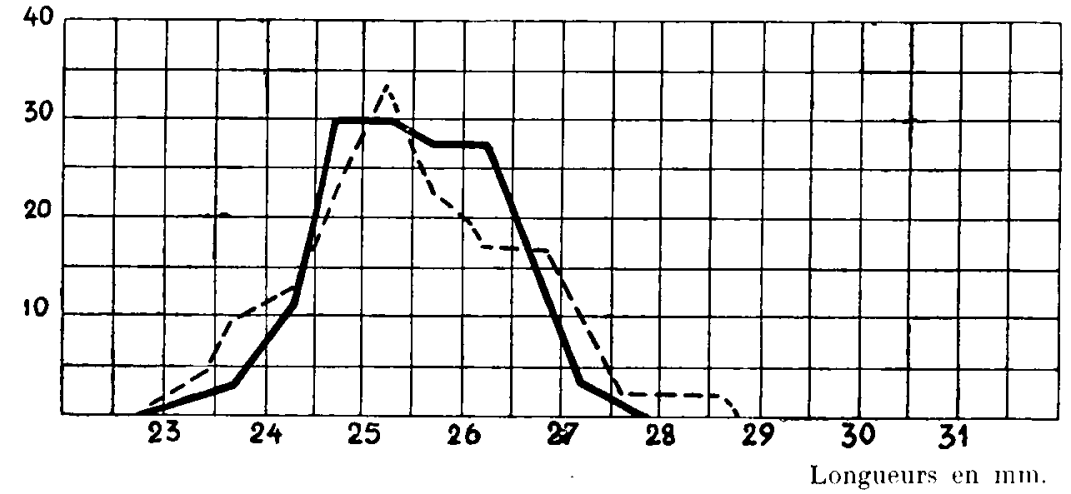

Fici. 14. - Courbe des longueurs, 13 zours apres la fécondation, 22 jours apres le début du nourrissage (d'apres Willerj.

- dans lange $\mathrm{C}$, obsiure. - dans lauge I), claire.

Quant à la mortalité pendant la durée de l'expérience (du 2 Décembre au ? Ir il), elle demeura loujours plus forte dans lauge iclairé ; pour les ceufs, elle fut de $2,6 \%$ dans $\mathrm{C}$ contre $0,66 \%$ dans $\mathrm{D}$; pour les alevins elle attcignait $27,3 \%$ contre sculement $1,33 \%$ dans le bassin obscur ; elle eut son maximum dans $C$, après le début du nourrissagce, alors que les conditions de croissance dans les deux augres étaient voisines.

On peut donc conclure que la lumière :

$1^{\circ}$ : - aucmente la mortalité des o'ufs ef surtout des alevins, principalement après le début de l'alimentation artificielle ;

$2^{0}:-$ ralentit la croissance surtout asant le díbut du nourrissagere.

L'action de la lumière est done néfaste et il est recommandé de couvrir les auges d'alevinage de pamneaux de bois ou de métal.

Les expériences précétentes avaient été failes dans des auges pauvres en oxygène $(2,7$ (entimètres cubes à 3,5 (contimètres cubes), mais tout de mêmẹ 
assez. loin des teneurs minima pour lalevin arant nourrissage que nous avons indiquées plus haut. Il était intéressant d'expérimenter dans le voisinagre de ces teneurs; aussi WiLLya (1), en opérant dans ces conditions limite; de vie, essaya de délerminer si le facteur "oxygène "l'emportait sur le facteur " lumière " et était, en quelque sorte, susceptible de le corriger; il répartit par moitié goo ceuf́s de Truites issus de même paren1s, le jour de leur fécondation ( 12 Korcmbre i9?7) dans deux auges différemment oxygénisées et éclairées, l'une ne contenant que I, 7 à I, 8 centimètrcs cubes d'oxygène (auge $\mathbf{I}$ ), mais maintenue à la lumière, l'autre, plus riche, en dosant I,9 à 2,3 centimètres cubes, mais restant obscure (auge II). Pen après l'embryonnement, le 7 Janvier 1928 , il préleva dans chaque bassin 225 wufs pour d'autres essais que nous examinerons tout à l'heure. Comment avaient évolué les $/ \bar{y} 0$ oufs restant, lorsque le 26 Février l'expérience fut arrêtée ? Dans l'auge $I$, obscure et pauvre en oxygène :

$1^{\circ}$ : - la mortalité avait été plus ronsidérable : ro alevins seulement vivants à la fin de l'expérience, alors que dans l'auge II il en subsistait ar.

Le manque de lumière n'avail pas corrigé l'action néfaste du manque. d'oxygène;

$2^{\circ}$ : - la durée de l'éclosion s'élail montrée beaucoup plus lonque (os jours contre 7 dans l'autre auge).

On a vu précédemment que l'obscurité seule avait une action de ce genre, mais beaucoup plus faible.

$3^{\circ}$ : - la croissance était fortemenl relardée : le 22. Février les huit survivants de cette auge ne mesuraient en moyenne que I,, 56 millimètres, crux de l'auge II alteignant, pour une moyenne de io survivants, 22,06 millimètres; les premiers avaient un poids moyen de 77 milliogrammes, les scronds de 94 milligrammes; il ne s'agrissait là que d'un retard, dû̀ à la l:uncue période d'ćclosion. Si l'on comparaît, on effet, dans les deux augres. le poids des alevins non plus le même jour mais au même stade de leur vie, par exemple à la fin de l'ćclosion (20 Février 19:8 dans I, 28 Février $19^{2} 8$ dans II), on trowait, au contraire un avantage en faveur de la première de ces augres; les rares subsistants y ćlaient mème légèrement plus grros, les chétifs n'ayant pu résister au manque d'oxyçène, mais ils avaient sur les individus de lautre auge un séricux retard à rattraper.

Les différences d'éclairement ont-elles une influence indépendante de l'olat d'évolution des ceufs ou des alevins? Wrderer, qui s'était posé la question, tenta de la résoudre dans une troisième série dexpériences que roici: le 7 Janvier rg98, on l'a vu, il avait prélevé, quelques jours après l'embryonnement, un lot de 2.55 ceufs dans l'auge I et une mème quantité dins l'aucre II. I.e prélèvement I, l'un bassin obscur, fut mis dans une auge III éclairée, au contraire du prélèvement II qui passa, dans une

(1) Op. cil., pp. 593 à 606. 
auğe IV, de la lumière à lobscurité; les deux nowvelles augres avaient unc teneur en oxygène voisine des aug̣es $I$ et $I I$ ( $I, 5$ à a centimètres cubes).

$I^{\circ}$. L Les cenfs de lauge III chaire, qui avaient conmencé leur évolution dans l'obscurité, se sont comportés comme ceux de l'auge I, maintenus constamment à l'abri de la lumière : le a l Févier igos, à la fin de l'éclosion qui dura près d'un mois, il ne restait que I I survisants. Ies oufs de l'auge IV obscure qui avaient été d'abord solimis à l'action de la lumière ont eu une évolution semblable à ceux de l'auge II ; le nombre des survivants à la fin de l'ólosion était de 200 : celle-ci n avait duré que 6 jours:

$x^{\circ}$. - L'éclosion fut avancée de 3 jours daus l'auge III par rapport à laugre J, tandis que dans les bassins II ef IV elle commengait à la meme late; le fait doit ctre altribú à ume richesse en oxygène lógèemement plus grande dans l'auge IIJ à ce noment-là (2 centimètres cubes): l'action de l'éclairement a été nulle ;

$3^{\circ}$. - Les alevins issus de cette éclosion variaient suicant leur provomane; ceux des auges I et III (movenno de 7 survivants) mesuraient les mèmes longueurs (24,70 millimètres et $24,9.3$ millimètres, le on Vars rg28), landis que ceux des augres $I I$ ef $I I$ adcusaient en leur faveur des différences $(97,08$ millimètres et $25,8 \mathrm{r}$ millimètres sur une moyenne de jo individus). L'auge II étant plus oxygénée que l'auge IV, res différences s'explicuent facilement.

Fn résumé, pour des eaux pauvres en oxygcine, l'action de ce gaz, avant l'embryonnement. l'emporte sur celle de la lumière: elle conditionne l'évolution de la croissance de l'alevin.

Examinons maintenant ce qui se passe lorsque les facteurs favorisant ou retardant la croissance sont surajoutés : une auge $X$ titrant de 3,9 à $7, \overline{\text { cecen- }}$ timètres cubes par litre, donc assez riche en oxygène, était maintenue à labri de la lumière (addition de deux facteurs favorisants); une augre $\mathrm{Y}$ ne contenant (que de $r, 48$ à 2,36 centimètres cubes par litre, donc pauve en oxygène, était au contraire fortement éclairée (addition de deux facteurs de retard de croissance). La température dans les deux auges oscillait, dans le courant de l'année, entre 5,2 et $8^{\circ}$; le débit était le même que précédemment. Wicher y plaça, le I4 Décembre i 927 , joo oeufs en provenance d'une seule femelle, fécondés le mìme jour par la laitance d'un seul mâle.

La différence entre les deux peuplements fut considérable ; dans l'auğe Y :

$I^{\circ}$. - L Lapparition de l'embryomement était retardé de 7 jours alors quaucune différence navail été constatée dans les expériences précédentes ;

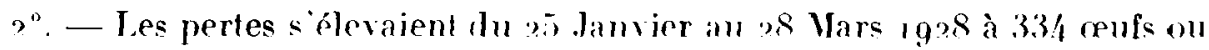
alevins, contre o dans l'auge $X$;

$3^{0}$. - Trois mois après la fécondation, le z 7 Mars, les alevins étaient moins 
longs et moins lourds dans des proportions considérables; ceux de l'auge $\mathbb{X}$ mesuraient $58 \%$ en plus et étaient plus lourds de 2. \%. Ces différences se continuèrent jusquà la fin de l'expérience, le 24 Mrril I928, où les individus de l'auge $Y$ mesuraient encore $25,-33$ millimètres contre 18,67 millimètres dans l'autre augre ;

$4^{\circ}$. - Les monstres élaient présents dans la proportion de $8 \%$ alors qu'ils n'existaient pas dans l'augre $X$.

La monstruosité parait dailleurs etre en relation avee une faible teneur en oxygène dans les derniers jours précédant l'éclosion, ainsi qu il résulte dautres observations de WILL.Er. Tous partagreons volontiers cette opinion.

En définitive, les actions ralentissantes, donc néfastes de la lumière el du manque d'oxygène sur la croissance des alevins sont bien élablies; aux salmoniculteurs d'agir en conséquence.

(A suivre).

\section{ERRATA}

Dans l'article intitulé : "Le Saumon revient-il à sa frayère natale ou, tout au moins, à son bassin d'origine? " inséré dans le précédent numéro, il y a lieu de rectifier ce qui suit :

Page 17, 10 ligne : substituer "six" à " cinq ".

Page 18, 23e ligne : substituer "kiloyrammes" à " kilomètres".

Page 18, 34" ligne : substituer " embouche " à " embouchure".

Page 19, 10" ligne : lire "Guipuzcoa " et non "Guiguscoa ".

Page $23,35^{\circ}$ et $36^{\circ}$ lignes : supprimer " non plus d'un bassin fluvial isolé mais d'un ensemble de bassins fluviaux interdépendants".

Page 25, $3^{\text {o }}$ ligne: Supprimer "pour". 